\title{
Synchronization between Background Activity and Visually Evoked Potential Is Not Mirrored by Focal Hyperoxygenation: Implications for the Interpretation of Vascular Brain Imaging
}

\author{
Stefan P. Koch, Jens Steinbrink, Arno Villringer, and Hellmuth Obrig \\ Berlin NeuroImaging Centre, Department of Neurology, Charité-Universitätsmedizin Berlin, 10117 Berlin, Germany
}

We performed an electroencephalography and optical topography study simultaneously exploring electrophysiological and vascular response magnitude as a function of stimulus frequency. To elicit a response in the visual cortex, subjects were exposed to flicker frequencies varying from 1 to $25 \mathrm{~Hz}$ ( $1 \mathrm{~Hz}$ steps, eyes closed). Extending the standard view to compare magnitudes of the evoked neuronal to the evoked vascular response, we additionally investigated modulations of $\alpha$-power, a marker of "background" EEG activity.

The results show two discrepancies between the electrophysiological and vascular response: (1) VEP and $\alpha$-power exhibit a discontinuous peak when stimulating at the individual $\alpha$-frequency (IAF) $(\sim 10-11 \mathrm{~Hz})$, indicating resonance between background oscillations and evoked response; this is not mirrored by the vascular response. (2) The vascular response, in contrast, steadily increases up to a maximum at $7-8 \mathrm{~Hz}$ and slightly decreases with higher frequencies. This continuous frequency dependence is partly reflected by the decrease in $\alpha$-power up to frequencies of $8-9 \mathrm{~Hz}$ and a slight increase in $\alpha$-power beyond the IAF resonance. Although indicating an inverse relationship between $\alpha$-power and vascular response, the frequency dependence of the evoked response does not show such a correlation.

Thus, electrophysiological resonance between an individual's $\alpha$-frequency and isofrequent stimulation is not mirrored by the vascular response. Also, spontaneous background EEG activity is an important modulator of the vascular response magnitude. We discuss these deviations from a simple one-to-one translation between evoked potential and vascular response amplitude in the light of questions concerning synchronization, attenuation, and induction of background oscillations such as the $\alpha$-rhythm.

Key words: flicker; steady-state visual-evoked potential (ssVEP); $\alpha$ oscillatory activity; hemodynamic response; near-infrared spectroscopy (NIRS); neurovascular coupling; visual cortex

\section{Introduction}

Beyond doubt, changes in neuronal activity are tightly coupled to focal changes in cortical blood flow. This constitutes the option to map the hemodynamic response and infer principles of the cortical processing, even of complex tasks (Villringer and Dirnagl, 1995). Nonetheless, recent research has highlighted the fact that the basis of such noninvasive brain imaging may be by far more complex because of fundamental deviations from a straightforward translation of neuronal excitation and inhibition into respective increases or decreases in vascular response (Mathiesen et al., 1998; Logothetis et al., 2001; Hewson-Stoate et al., 2005).

Here we highlight a related issue, dealing with the fact that "at rest" the brain entertains an impressive electrophysiologically ac-

\footnotetext{
Received Sept. 20, 2005; revised March 9, 2006; accepted March 31, 2006.

This work was supported by the Bundesministerium für Bildung und Forschung. The technical equipment was made possible by the Europäischer Fond für regionale Entwicklung.

Correspondence should be addressed to Stefan P. Koch, Berlin Neurolmaging Centre, Department of Neurology, Charité-Universitätsmedizin Berlin, Campus Mitte, Schumannstrasse 20-21, 10117 Berlin, Germany. E-mail: stefan.koch@charite.de.

DOI:10.1523/JNEUROSCI.3989-05.2006

Copyright $\odot 2006$ Society for Neuroscience $\quad$ 0270-6474/06/264940-09\$15.00/0
}

cessible activity, which is modulated and/or superimposed by an evoked response (Makeig et al., 2002). [For the complex relationship between intracortical and scalp-recorded electrophysiological potentials, see Mitzdorf (1985) and Lopes da Silva (2004)]. Because modulation of such "background" activity has been shown to elicit regional blood oxygenation level-dependent (BOLD) changes (Goldman et al., 2002; Laufs et al., 2003; Moosmann et al., 2003), one motivation for our study was to modulate both spontaneous oscillations and evoked potential amplitude in a parametric visual stimulation design, thus investigating their respective influence on regional cerebral blood flow ( $\mathrm{rCBF}$ ) changes. To this end, we examined a flicker-light stimulation over a wide frequency range, while subjects kept their eyes closed. Separating the amplitude of the visual-evoked potential (VEP) from $\alpha$-power modulation, two potential predictors of the simultaneously assessed vascular response can be analyzed.

Another goal of the study was to address a surprisingly neglected, although striking discrepancy between the frequency dependence of the vascular response and VEP amplitude. Vascularbased techniques report on a continuously differentiable increase in response up to $\sim 8 \mathrm{~Hz}$ (Fox and Raichle, 1985; Kwong et al., 
1992; Mentis et al., 1997; Ozus et al., 2001), whereas a discontinuous peak of the VEP amplitude at stimulation frequencies around the individual $\alpha$ frequency (centered at $\sim 10 \mathrm{~Hz}$ ) have long been reported (Herrmann, 2001). Imaging studies may have "missed" the discontinuity around the individual $\alpha$-frequency because of a rather coarse modulation of the stimulation frequency (Pastor et al., 2003), whereas electrophysiological data are available between 1 and $100 \mathrm{~Hz}$ in $1 \mathrm{~Hz}$ steps (Herrmann, 2001). Therefore, we investigated frequencies between $5-25 \mathrm{~Hz}$ in $1 \mathrm{~Hz}$ steps and normalized our results to the individual IAF. We expect that the discontinuous peak in VEP amplitude when stimulating at the IAF is not mirrored by a similar peak amplitude in the oxygenation changes as a measure of the vascular response. This will have implications for the relationship between ongoing and evoked electrophysiological activity: because the vascular response reflects the integrated afferent signaling and intracortical processing (Lauritzen, 2001; Attwell and Iadecola, 2002), a resonance between evoked potential and $\alpha$-rhythm without a correspondingly larger vascular response supports the view that the evoked potential is largely generated by synchronization and phase locking.

Thus, although reports on deviations from a straightforward neurovascular coupling might falsely increase a general skepticism in the validity of vascular-based imaging techniques, we demonstrate that, in contrast, such deviations disclosed by combined approaches may even help to address questions of synchronization and summation of neuronal signaling, formerly a domain of electroencephalographic techniques (Pareti and De Palma, 2004).

\section{Materials and Methods \\ Experimental procedure}

Because the hypothesis of the study relies on the differentiation between the evoked electrophysiological response [steady-state visual-evoked potential (ssVEP)] and spontaneous background activity (represented by $\alpha$-power), we chose a flicker-light stimulation while subjects kept their eyes closed. $\alpha$-Modulation and evoked potential amplitude dependency on parametric frequency modulation were thus accessible.

\section{Stimulation protocol}

Subjects were comfortably seated in an EEG chair, in a silent, dark room wearing goggles to apply visual stimulation at the frequencies of $1 \mathrm{~Hz}$ and between 5 and $25 \mathrm{~Hz}$ in $1 \mathrm{~Hz}$ steps. These 22 different frequencies were presented in trials of $15 \mathrm{~s}$ duration each. The full experiment consisted of six blocks, each of which included one trial of each frequency and three trials without stimulation (resting periods). Within a block, the different frequencies and resting periods were presented in a pseudorandom order without intertrial intervals. Between blocks, the subject was allowed a break to prevent fatigue. This is mandatory, because relaxed wakefulness should be maintained during the full duration of the experiment lest to alter spontaneous and evoked responses by varying levels of alertness. Each stimulus consisted of a $5 \mathrm{~ms}$ rectangular red light pulse $(9000 \mathrm{mcd}$ at $600 \mathrm{~nm}$ ) generated by two light-emitting diodes (Conrad Electronic, Berlin, Germany) in binocular goggles, equivalent to a full visual field stimulation. The pseudorandom order of the stimuli in trials of $15 \mathrm{~s}$ was programmed with the software Presentation (version 0.71; Neurobehavioral Systems, Albany, NY). Stimulation frequency was determined by the interval between flashes, i.e., for the highest frequency $(25 \mathrm{~Hz})$, the 5 $\mathrm{ms}$ flashes were separated by $35 \mathrm{~ms}$ intervals, whereas at $1 \mathrm{~Hz}$ stimulation they were separated by $995 \mathrm{~ms}$ (Fig. 1b).

In summary, we acquired six trials for each of the 22 different frequencies and 18 trials without stimulation. All trials had a duration of $15 \mathrm{~s}$. The experiment lasted $<45 \mathrm{~min}$ including the subject-guided breaks between stimulation blocks. Montage of EEG and near-infrared spectroscopy (NIRS) probes required $\sim 30-40$ min before the experimental procedure was started.



Figure 1. Set-up and experimental design. $\boldsymbol{a}$, The positioning of the electrodes (open gray circles) according to the 10-20 system for the positions $01,02, \mathrm{P3}, \mathrm{P} 4$, and Pz. The array of the NIRS topography system is given by the positions of the light-emitting (black circles) and -detecting (white circles) probes, whereas the approximate sampling volume is sketched by the connecting ellipses. The inlay shows how the optical probes at 01 and 02 are inserted in the EEG ring electrodes, allowing for an optimal coregistration between EEG and NIRS. $\boldsymbol{b}$, A sketch of the stimulus design. The 22 different frequencies are presented in trials of $15 \mathrm{~s}$ duration in a pseudorandom order. The enlargement shows an example for the $20 \mathrm{~Hz}$ condition consisting of $5 \mathrm{~ms}$ flashes and an interflash interval of $45 \mathrm{~ms}$. For a stimulation frequency of, e.g., $10 \mathrm{~Hz}$, the duration of the flash (5 ms) remained constant, and the interflash interval was $95 \mathrm{~ms}$ (data not shown).

\section{Subjects}

From an initial EEG study (without coregistration of NIRS; data not reported here), we selected 11 of 21 subjects, who showed a well detectable $\alpha$-rhythm. None of the thus selected subjects had to be excluded from additional analysis in the here reported combined EEG-NIRS study [2 male, 9 female; mean age, 25.4 years (range, 18-28 years)]. All subjects had normal vision or mild hyper-/myopia, were neurologically and otherwise healthy with unremarkable medical history (especially no migraine or epileptic seizures). Correction of mild hyper-/myopia was not mandatory because full-field stimulation was applied while eyes were closed. All subjects gave informed consent and were financially rewarded for their participation. Beyond tiring, none of the subjects reported any major discomfort.

\section{Data acquisition}

EEG. To assess $\alpha$-power and ssVEP, the EEG (BrainAmp amplifier and Vison Recorder software; Brain Products, Munich, Germany) was recorded over 21 standard positions according to 10-20 system (Fp1, Fp2, F3, F4, C3, C4, P3, P4, O1, O2, FC1, FC2, CP1, CP2, FC5, FC6, CP5, CP6, $\mathrm{Fz}, \mathrm{Cz}, \mathrm{Pz}$ ). Three additional channels recorded the horizontal and vertical oculogram $\left[\mathrm{hEOG}_{\mathrm{L}}, \mathrm{hEOG}_{\mathrm{R}}, \mathrm{vEOG}_{\mathrm{U}}\right.$ (left horizontal, right horizontal, and vertical upper electro-oculogram, respectively)]. During recording, all electrodes were referenced to $\mathrm{FCz}$, and data were recorded at a $1000 \mathrm{~Hz}$ sampling frequency $(0.3-70 \mathrm{~Hz}$ frequency range with $50 \mathrm{~Hz}$ notch and $3 \mathrm{~s}$ amplification).

Optical imaging. As an indicator of the vascular response, we assessed changes in hemoglobin oxygenation measured over the occipital region using a frequency-domain NIRS imaging system, based on a multichannel Omniat Tissue Oxymeter (ISS, Champaign, IL). We here focus on the decrease in deoxy-hemoglobin, which can be only explained by a faster washout during an increase in regional cerebral blood flow. Also, the content of [deoxy- $\mathrm{Hb}]$ is the most relevant physiological parameter determining the BOLD contrast. A decrease in [deoxy- $\mathrm{Hb}$ ] is correlated to an increase in BOLD contrast (Kleinschmidt et al., 1996). Details of the methodology and the underlying physiology were detailed previously (Obrig and Villringer, 2003). In brief, light in the near-infrared penetrates biological tissue rather well, thus allowing for optical spectroscopy in deeper tissue layers such as the cerebral cortex. Changes in oxygenation of hemoglobin elicit a change in absorption at different wavelengths, broadly corresponding to the well known color change of venous and arterial blood in the visible range. Using two wavelengths and applying a modified Beer-Lambert law, concentration changes in oxygenated and deoxygenated hemoglobin can be thus assessed $(\delta[\mathrm{oxy}-\mathrm{Hb}]$ and 


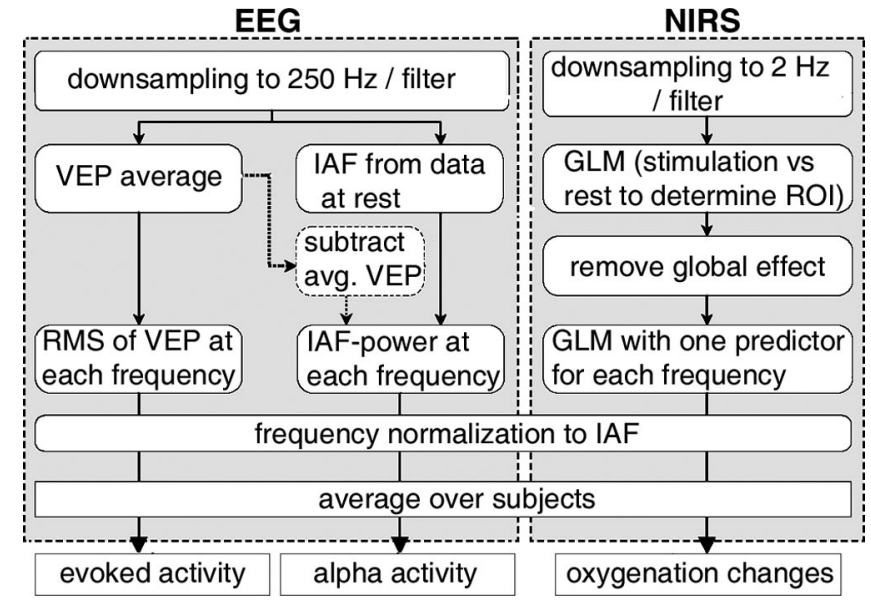

Figure 2. The flow chart illustrates the analysis of EEG and NIRS data. NIRS: After filtering and downsampling, a GLM was applied to determine the pixels (ROI) in whom the difference between rest and stimulation at any frequency was most significant. Next, an attenuation of global effects was performed (see Materials and Methods). For the time courses in the ROl, we performed a full GLM with 22 predictors (one predictor per frequency). EEG: After downsampling and filtering, the IAF and the mean VEP for each frequency was determined. The RMS of $1 \mathrm{~s}$ periods was assessed to yield a measure of the amplitude of the evoked response. To assess $\alpha$-power, two analyses were performed. The PSD at the IAF was determined for each frequency without and after (broken arrow) an attenuation of the stimulus-evoked response. The attenuation was done by subtracting the respective mean SSVEP time-locked to each individual stimulus in the time domain. The PSD was then processed as for the non-stimulus-attenuated data (for the different results, compare Fig. $4 b$, open vs filled circles).

$\delta[$ deoxy-Hb]) (Cope and Delpy, 1988). Our system uses laser-diodes emitting light at 690 and $830 \mathrm{~nm}$, respectively. The intensity of the incident light is modulated at $110 \mathrm{MHz}$. Guided to the subject's head by fiber-optic bundles (diameter, $0.4 \mathrm{~mm}$ ), some of the light will be reflected and can be collected by another optic probe (diameter, $3 \mathrm{~mm}$ ) some centimeters apart, to guide the light to the detector (photon multiplier tube). The resulting changes in the intensity and changes in the phase shift of the modulation wave allow a description of changes in optical density in the illuminated tissue [the option of depth resolution (KohlBareis et al., 2002) was not applied here]. Assuming constant scatter, these changes can be converted into changes in [oxy- $\mathrm{Hb}$ ] and [deoxy$\mathrm{Hb}$ ] in the tissue illuminated. To provide a rough topographical image, the system uses seven emitter and four detector channels arranged in an array covering $50 \mathrm{~cm}^{2}$ of the occiput. The center of the probe array was 2 $\mathrm{cm}$ above the inion. Thus, the array covers the area around electrodes $\mathrm{O} 1$ and $\mathrm{O} 2$. The position of the optical probes in relation to the electrodes is demonstrated in Figure 1a. Source-detector distance according to the position in the EEG cap was $2.5 \mathrm{~cm}$. Because the cap is stretched to suit the individual subject, this distance varied slightly between subjects (2.3$2.7 \mathrm{~cm}$ ). Within one subject, the interprobe distance was constant. Data were recorded at $10 \mathrm{~Hz}$.

\section{Data analysis}

Data were analyzed with programs written in Matlab in part using preprogrammed routines (version 6.5; The Mathworks, Natick, MA). For all analyses concerning frequency dependence of the stimulus-locked activity and $\alpha$-activity, data were derived from electrode $\mathrm{O} 2$. Because the detailed description of the different steps of our analysis of EEG and NIRS data may be beyond some readers' interest, we supply a simple flow chart of the data processing in Figure 2. In summary, we performed the analysis to yield frequency dependencies of (1) the evoked electrophysiological response (VEP/ssVEP); (2) an indicator of the ongoing electrophysiological background activity ( $\alpha$-power) and (3) the vascular response as indicated by the hemoglobin-oxygenation changes. Because the IAF differs between subjects, we normalized the frequency dependencies to the IAF.

\section{EEG}

Evoked activity. For reasons of computational velocity and because gamma oscillations are not a focus of the present study, data were downsampled off-line with Vision Analyzer software (Brain Products) to 250 $\mathrm{Hz}$ (using a cubic spline interpolation, third-order polynom). To assess amplitude of the visual-evoked potentials, we calculated VEP at $1 \mathrm{~Hz}$ and ssVEP $(5-15 \mathrm{~Hz})$ for each stimulation frequency at all electrode positions. Beginning with the third stimulation within a stimulation trial, time windows of $1000 \mathrm{~ms}$ starting at each succeeding stimulus were averaged for all electrodes. The results of the six trials of identical stimulation frequency were averaged to periods of $1 \mathrm{~s}$. As a measure for stimulus-locked evoked activity, the root mean square (RMS) of these periods was determined. The alternative, to assess peak amplitude of a single VEP for each frequency to then multiply by frequency (Arthurs et al., 2000), yielded similar results and is not reported here.

Background activity. To estimate the $\alpha$-activity, all stimulation trials were analyzed in the frequency domain by an estimate of the power spectral density (PSD) with a window $\left(n_{\mathrm{FFT}}\right)$ of 1000 data points (corresponding to $4 \mathrm{~s}$ ), using a Hanning window with an overlap of half the $n_{\mathrm{FFT}}\left(n_{\text {overlap }}=500\right.$ data points $\left.=2 \mathrm{~s}\right)$. Segments were linearly detrended before the fast Fourier transform (FFT) was applied. For each subject, the mean IAF was determined by the PSD estimate of the 18 resting trials at electrode O2. Changes in $\alpha$-power were estimated for each stimulation frequency with reference to the PSD at the IAF taken from the resting trials. This rather basic analysis of determining the $\alpha$-activity at different stimulation frequencies introduces a problem of indifference: when the stimulation frequency converges to the subject's IAF, it is not possible to segregate $\alpha$-activity from evoked activity locked to the stimulus. Therefore, we additionally performed an attenuation of the evoked response:

\section{VEP subtraction}

To better differentiate between stimulus-locked electrophysiological activity (ssVEP) and the background activity represented by $\alpha$-power, stimulus-locked activity was attenuated in each stimulation period before the above procedure: for each subject, each frequency, and all electrodes, the corresponding mean VEP was subtracted after each stimulus in the raw data. Thus, the respective mean stimulus-induced activity was attenuated in the time domain before the estimate of $\alpha$-activity along the spectral analysis procedure described above. There may be some doubt whether this attenuation procedure will introduce a confound because a periodic stimulus is applied. Therefore, we tested our algorithm in a simulation. The results prove that even with high levels of trial-to-trial variability, the attenuation procedure does not introduce a systematic confound. The simulation results are available as supplemental material (at www.jneurosci.org).

\section{Optical imaging}

The vascular response was assessed by the analysis of changes in [deoxy$\mathrm{Hb}]$ and $[\mathrm{oxy}-\mathrm{Hb}]$ as measured by near-infrared spectroscopy. Because changes in [deoxy-Hb] correspond to BOLD contrast changes as assessed by functional magnetic resonance imaging (fMRI) (Kleinschmidt et al., 1996), the forthcoming analysis focuses on [deoxy-Hb]. After calculation of the concentration changes in the concentration of hemoglobins, we attenuated the pulse-related signal changes by a lowpass filter (Butterworth, fifth order) at $0.5 \mathrm{~Hz}$. Optical images were generated over a $\sim 50$ $\mathrm{cm}^{2}$ area overlying the occipital cortex by interpolation of the 14 emitter-detector combinations measured (Fig. 1a).

The additional analysis comprised a number of successive steps.

Selection of "activated pixels." The first step was performed to determine the pixel with the most significant difference between resting periods and stimulation. Thus, all trials with stimulation $(1 \mathrm{~Hz}$ and $5-25 \mathrm{~Hz})$ were considered the on-condition, whereas resting periods $(n=18)$ served as off-condition. In analogy to statistical parametric mapping (e.g., Statistical Parametric Mapping software SPM99; Wellcome Department of Cognitive Neurology, London, UK), the predictor (boxcar of on-condition vs off-condition) was convoluted with a negatively accelerated exponential function (gamma function). The inverse response direction stems from the fact that a decrease in [deoxy-Hb] corresponds to an increase in BOLD contrast. Statistics followed $\beta$-value estimates along the principles of the general linear model (GLM). 
Attenuation of "global effects." In a second step, global effects were attenuated across all measurement positions. The hypothesis is that such effects are likely to stem from movement artifacts or from hemodynamic changes unrelated to the stimulation (breathing, low frequency oscillations, slow changes in blood pressure, and residual heart beat). Because our prediction is that the global effects are not correlated with the stimulation, we chose those six positions with the smallest $t$ values extracted from the first analysis. Those time series were then fed into a principal component analysis (PCA). Note that only six pixels where used, to prevent an attenuation of the stimulation-induced changes. The resulting orthogonal time courses were used as covariates in the subsequent analysis.

Frequency dependence. To compare [deoxy-Hb] changes for the different stimulation conditions (frequencies), the input response function for each frequency was convoluted with the hemodynamic response function (i.e., predictors for each stimulation frequency were determined). Finally, all predictors and the covariates (as obtained by the PCA) were integrated in the GLM analysis. For each subject, the location with the largest $t$ value was selected to determine the dependence of [deoxy- $\mathrm{Hb}$ ] changes from the stimulation frequencies.

Before averaging across subjects, we normalized the individual subject's data with respect to frequency and amplitude.

Frequency normalization. Frequencies of the human EEG can be expressed as units relative to the individual central $\alpha$-frequency. Because we here focus on stimulation-induced modulation of $\alpha$-rhythm, we performed a frequency normalization. For example, in a subject with an IAF of $8 \mathrm{~Hz}$, the $5 \mathrm{~Hz}$ stimulation corresponds to a stimulation at $0.63^{\star} \mathrm{IAF}$, whereas the stimulation at $25 \mathrm{~Hz}$ corresponds to $3.13^{\star} \mathrm{IAF}$. In a subject with an IAF of $12 \mathrm{~Hz}$, correspondingly, frequencies from $0.42^{\star} \mathrm{IAF}$ to $2.08^{\star} \mathrm{IAF}$ were tested. This conversion is based on the underlying hypothesis that biological response will depend on the biological frequencies rather than on absolute numeric values.

Amplitude normalization. For each subject, the frequency-normalized data of all parameters were $z$-scored using the formula $Z i=(P i-$ mean $(P i)) /$ std $(P i)$, where $P$ means the data of the particular parameter for the subject $i$. Next the $z$-scored data were averaged across subjects. Finally, we retransformed data to absolute values conserving mean and variance of the original data. For an example of the normalization procedures, see supplemental material, available at www.jneurosci.org.

\section{Results}

The central issue of the present paper is the differentiation between evoked electrophysiological activity (ssVEP) and spontaneous background activity to investigate their respective influence on the vascular response. This section first presents the ssVEP results to then describe stimulation-frequency dependent changes in $\alpha$-power. The latter is considered the most prominent feature of electrophysiological background activity over the occipital cortex. Finally, the results of the NIRS measurements are shown to yield a tentative hypothesis on the mutual dependence of the two electrophysiological predictors and the vascular response. It should be noted that evoked or induced $\gamma$-power changes are another intriguing oscillatory electrophysiological signal, which has been discussed with respect to the vascular response (Niessing et al., 2005). We did not find any reliable modulation in the $\gamma$-band, which may stem from the "stationary" nature of the stimulus (Sewards and Sewards, 1999) but may likewise result from a low signal-to-noise ratio. Therefore, we here focus on the $\alpha$-band changes, which were clearly modulated in all subjects.

As described in Materials and Methods, we normalized the frequency dependence to the individual mean $\alpha$-frequency derived from the resting periods. This procedure results in different scales for each subject, resulting from the interindividual difference in $\alpha$-frequency. Also, because the study design only included integer steps of $1 \mathrm{~Hz}$ for the stimulation frequencies, a stimula- tion at the exact $\alpha$-frequency was usually not available. Therefore, after averaging, we rescaled the stimulation frequency range to integer frequencies (in hertz) by multiplying with the mean $\alpha$-frequency across all subjects $(10.6 \mathrm{~Hz})$. Thus, the presentation of the results gives data at frequencies that were actually tested. The mean $\alpha$-frequency across subjects and its harmonic are indicated by broken bars in the plots. In the text we refer to frequencies in hertz, which can be converted into multiples of the IAF by division by 10.6 .

\section{Electrophysiological results}

ssVEPs

VEP or ssVEP traces for all frequencies are shown in Figure $3 a$ (the transition from a separable VEP to a steady-state oscillation is somewhat arbitrary. In a strict sense, only the result at $1 \mathrm{~Hz}$ can be considered a typical VEP, because at $5 \mathrm{~Hz}$, components with a latency $>200 \mathrm{~ms}$ will be aliased and cannot be differentiated). They are a grand average across data at electrode position O2 from all subjects and all stimulation blocks. After $z$-transformation (across stimulus frequencies) of the individual subjects, the averaged data were rescaled to microvolts by multiplication of the mean amplitude across subjects. For low stimulation frequencies $(1,5$, and $6 \mathrm{~Hz})$, two relatively distinct early components with latencies of $\sim 80$ and $\sim 110$ ms can be discriminated. These components broadly correspond to the N2 and P2, as defined in clinical VEP guidelines for flash-evoked VEP (Odom et al., 2004). From 7-9 Hz, the ssVEP shows a biphasic response, without clearly discernable components to yield roughly sinusoidal oscillations beyond $9 \mathrm{~Hz}$. As a measure of mean electrophysiological activity, the root-mean square (RMS) of stimulus-evoked potentials was assessed over the $1 \mathrm{~s}$ data segments. In Figure $4 a$, this measure of mean amplitude and SEM across subjects is plotted against stimulus frequency. The stimulus-evoked response is largest in amplitude at 5 and $11 \mathrm{~Hz}$, and a local minimum can be seen at $7 \mathrm{~Hz}$. Beyond $11 \mathrm{~Hz}$, evoked responses decrease in amplitude with increasing stimulation frequency. Small local maxima appear at 20 and $25 \mathrm{~Hz}$.

\section{$\alpha$-Power modulation}

As described in Materials and Methods, we performed two types of analysis to assess the dependence of $\alpha$-power from the stimulus frequency applied. Results for either analysis are presented in Figure $4 b$. The open symbols show the frequency dependence of $\alpha$-power, when applying the conventional method. There is a clear peak around the mean $\alpha$-frequency. Necessarily, stimulusevoked response and $\alpha$-frequency spectrally coincide at this frequency. Thus, the result cannot be easily interpreted with respect to the underlying question of the mutual interdependence between electrophysiological background activity and vascular response. Therefore, for the second type of analysis, we subtracted mean ssVEP from the raw data before the analysis in the frequency domain. The results for this analysis are given by the black symbols. In addition to the attenuation of the peak at the mean $\alpha$-frequency, there is another relevant difference between the results of the two analyses. At $\sim 5 \mathrm{~Hz}$, the type I analysis shows a "subharmonic" peak in $\alpha$-power not seen after attenuation of the evoked response. Note that the peak at $\sim 21-23 \mathrm{~Hz}$ is not altered by the stimulus attenuation procedure.

To sum up, $\alpha$-power decreases with stimulus frequency to reach a minimum at $9 \mathrm{~Hz}$. At the individual $\alpha$-frequency, there is a clear increase in $\alpha$-power, which is larger than the resting $\alpha$-power but nearly identical to resting $\alpha$-power after subtraction of mean ssVEP (Fig. 4b, filled symbols). Beyond $11 \mathrm{~Hz}, \alpha$-power 




Figure 3. Grand-average time courses for ssVEPs and [deoxy-Hb] changes. $\boldsymbol{a}$, The visualevoked potentials averaged across all subjects. Segments of $1 \mathrm{~s}$ were averaged for all frequencies tested. The stimulation frequency is given on the left in hertz; vertical bars denote the flashes, horizontal lines denote $0 \mu \mathrm{V}$ for each frequency. Note that only the first $330 \mathrm{~ms}$ of each frequency are presented for reasons of graphical clarity. The resonance at $\sim 11 \mathrm{~Hz}$ and more weakly at $\sim 21 \mathrm{~Hz}$ can be seen. $\boldsymbol{b}$, The time course of the [deoxy- $\mathrm{Hb}$ ] changes in response to the 22 different frequencies tested. The gray vertical box indicates the stimulation epoch. The decrease in [deoxy-Hb] is an indicator of an increase in cerebral blood flow (washout of [deoxy$\mathrm{Hb}]$ ). Larger decreases thus denote a larger vascular response. Error bars denote SEM. Note that in contrast to $\boldsymbol{a}$, giving the sSVEPs, the whole stimulation epoch (15 s) is shown, because the vascular response is too sluggish to analyze individual stimulus response.



Figure 4. Frequency dependence of the electrophysiological parameters. $\boldsymbol{a}$, The average magnitude of the evoked potentials, calculated as the root mean square over the $1 \mathrm{~s}$ segments (as shown, in part, in Fig. 3a). $\boldsymbol{b}$, The frequency dependence of the $\alpha$-power before (open circles) and after (filled circles) attenuation of the stimulus-induced changes (for the procedure, see Materials and Methods and Fig. 2). Error bars give the SEM across subjects. Note that all data were normalized to the IAF before averaging. The data were transformed back to actually tested frequencies by multiplying with the mean IAF across subjects of $10.6 \mathrm{~Hz}$. The vertical bars give the mean $\alpha$-frequency $(10.6 \mathrm{~Hz})$ and its first harmonic $(21.2 \mathrm{~Hz})$. The horizontal solid bar denotes resting state $\alpha$-power with SEM (dashed lines).

shows a tendency to increase with stimulus frequency. A broader peak in the $\beta$-frequency range $(21-23 \mathrm{~Hz})$ is not altered by the attenuation of stimulus-induced response, whereas at the peak at the subharmonic of $\alpha$, at $\sim 5 \mathrm{~Hz}$ is eliminated by the procedure.

\section{Hemodynamic response}

Hemodynamic response was assessed by measured changes in [deoxy-Hb] and [oxy-Hb]. Because changes in [deoxy- $\mathrm{Hb}$ ] inversely correlate with BOLD contrast as assessed in fMRI, the analysis is focused on this parameter. After selection of the focal maximum of stimulation-related decreases in [deoxy- $\mathrm{Hb}$ ] (see Materials and Methods), we analyzed the dependency of [deoxy$\mathrm{Hb}$ ] decreases from stimulation frequency. Note that a larger decrease in [deoxy- $\mathrm{Hb}]$ is interpreted as an indicator of an increase in blood flow velocity, it can only stem from an increased washout of deoxygenated hemoglobin from the volume sampled, because spontaneous reoxygenation of hemoglobin is specific to the pulmonary vascular bed. This can be termed "activation" in line with the terminology in studies using other vascular based methodologies [fMRI and positron emission tomography $(\mathrm{PET})]$. Figure 5 depicts the results for [deoxy-Hb] and [oxy-Hb] averaged across all subjects. Figure $5 a$ depicts the results for [deoxy- $\mathrm{Hb}$ ], which maximally decreases at stimulation frequencies of 7 and $8 \mathrm{~Hz}$. Lower frequencies elicit a smaller response, whereas beyond $8 \mathrm{~Hz}$, the [deoxy- $\mathrm{Hb}$ ] response is stable, showing a tendency to be slightly attenuated. The [oxy-Hb] changes (Fig. $5 b$ ) show a clear difference between stimulation of $1 \mathrm{~Hz}$ and all other stimulation frequencies; however, potentially to high variability, a clear maximum of the response at $7-8 \mathrm{~Hz}$ cannot be 


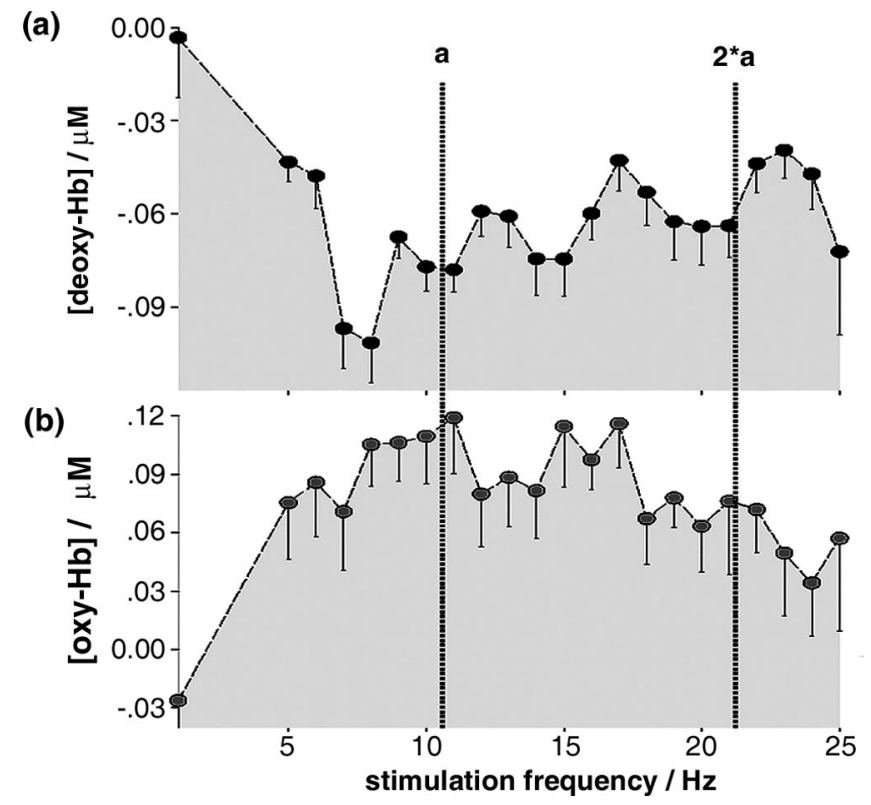

Figure 5. Frequency dependence of the hemodynamic parameters. Changes in [deoxy-Hb] $(\boldsymbol{a})$ and [oxy-Hb] (b) are given in analogy to Figure 4. The same normalization procedure was applied (IAF normalization and back-transformation). Mean $\alpha$-frequency and first harmonic are indicated by vertical bars.

seen. The reasons for differences between changes in [oxy-Hb] and $[$ deoxy-Hb] are complex but beyond the scope of the present paper. For a more detailed discussion on the flow volume relationship and its implications for NIRS-fMRI measurements, we refer to a publication to be issued shortly (Steinbrink et al., 2006).

In analogy to the mean ssVEPs, Figure $3 b$ depicts the averaged time courses for [deoxy-Hb] for all frequencies in units of hertz for a timescale of $30 \mathrm{~s}$. Because stimulation frequencies are not separated by resting periods, a reconstruction based on linear superposition instead of simple averaging was done. After onset of stimulation, [deoxy-Hb] decreases at all frequencies. As also seen in Figure $5 a$, [deoxy-Hb] reveals a maximal decease at stimulation frequencies of 7 and $8 \mathrm{~Hz}$.

Relationship between electrophysiological and vascular response The comparison between the two aspects of the electrophysiological response (i.e., the ssVEP and the $\alpha$-power) and, in contrast, the hemodynamic response, critically depends on the sampling of a comparable region of the cerebral cortex. A fine-tuned spatial correlation is beyond the spatial resolution of either method. EEG and NIRS can localize on a scale of centimeters rather than millimeters. Nonetheless, we considered it mandatory to provide arguments for the comparability of the two response modalities. Figure $6 a$ shows the topological distribution of visual-evoked potentials for all stimulation frequencies tested. The topological maps indicate that the largest potentials are located in the occipital region. The response was maximal over the leads $\mathrm{O} 1$ and $\mathrm{O} 2$. These two electrodes are at the center of the area investigated by the NIRS array. For NIRS, Figure $6, b$ and $c$, shows the $t$-maps of [deoxy-Hb] and [oxy-Hb] for the comparison resting periods versus all stimulation periods. This test was performed to select the channel in which the frequency dependence of the hemodynamic changes was analyzed. Figure $6 d$ shows the optical probe arrangement. Figure $6 e$ gives the location of the highest $t$ value of [deoxy-Hb] and [oxy-Hb] for each subject. The results show that the decrease in [deoxy-Hb] showed highest $t$ values (navy-blue (a)

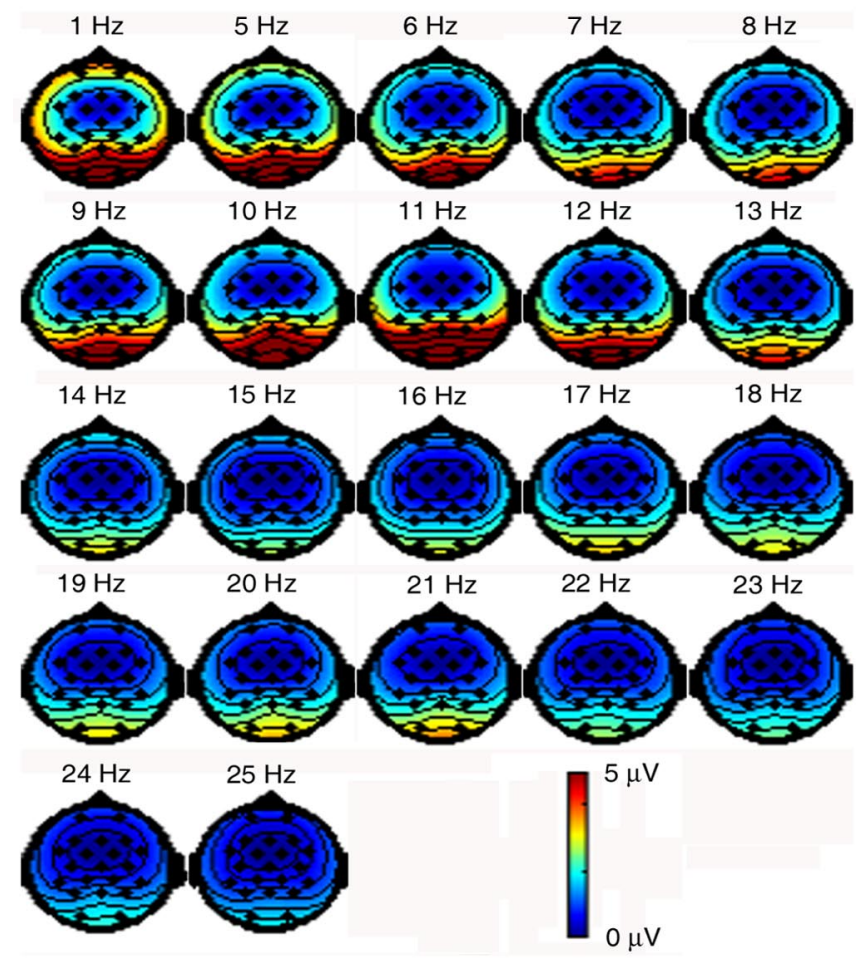

(b)

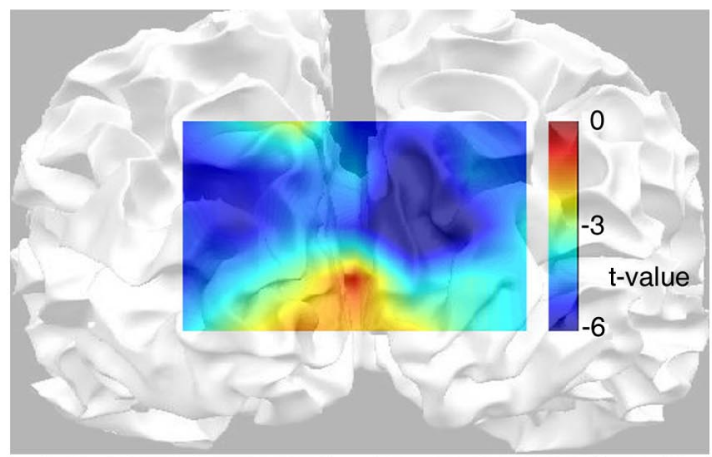

(c)

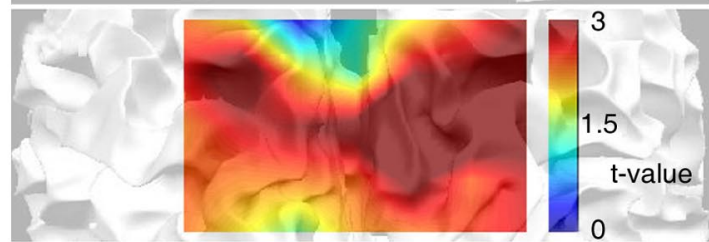

$(d+e)$

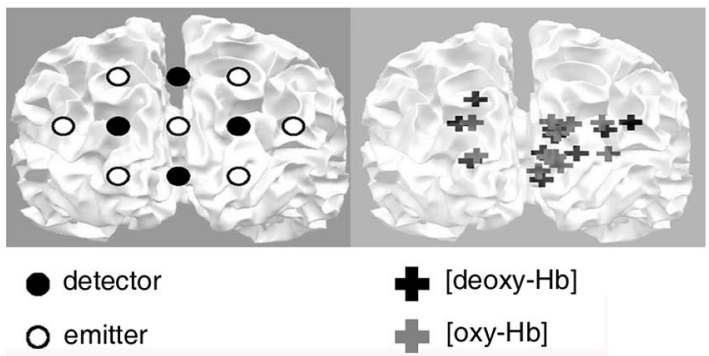

Figure 6. EEG and NIRS topography. $\boldsymbol{a}$, Spherical spline maps of the RMS of averaged visualevoked potentials on a scalp in a two-dimensional circular view. The topography is generated using interpolation on a fine Cartesian grid based on the measurement over the 21 standard 10-20 system positions. Maps for each flicker frequency tested are given. Pesudocolor scaling is in $\mu \mathrm{V}$ ). $\boldsymbol{b}$, Topographical pseudocolor $t$-maps of changes in [deoxy-Hb] and [oxy-Hb] (c). Note the generally higher $t$ values for [deoxy-Hb]. $\boldsymbol{d}$, The optical probe arrangement; $\boldsymbol{e}$, the location of the highest $t$ values for [deoxy-Hb] and [oxy-Hb] for each subject demonstrating the intersubject variability. 
areas in the pseudo-color scaling) for the area overlying electrodes $\mathrm{O} 1$ and $\mathrm{O} 2$. We decided to analyze the data in one electrode $(\mathrm{O} 2)$ to yield a comparable result with respect to the NIRS results based on a selected single probe pair. We applied a frequency normalization to sharpen the resonance phenomenon between $\alpha$-oscillation and ssVEP. To judge whether such an electrophysiological resonance would translate into a likewise augmented vascular response at this frequency, the same normalization was performed for the NIRS data. Un-normalized EEG data in fact showed the expected broadening of the resonance peak, whereas the vascular response parameters did not show any discontinuous increase around the central or normalized $\alpha$-frequency (data not shown). Correlation analysis was performed between [deoxy-Hb]/ssVEP and [deoxy-Hb]/ $\alpha$-power, respectively. This correlation analysis across all stimulation frequencies revealed no significant results for the group average. Nonsignificant correlations were also found in all but three comparisons in the single subjects (supplemental Table 1, available at www.jneurosci.org as supplemental material).

To sum up, evoked potentials and stimulation-induced hemodynamic changes were maximal over the occipital region roughly localized between $\mathrm{O} 1$ and $\mathrm{O} 2$, when relying on the 10-20 system. The simplifying hypothesis is that changes in regions outside this area will not substantially influence the evoked response elicited by the stimulus. Anatomically, the region sampled includes striate and extrastriate visual areas. The question of how far subcortical neuronal activity is relevant to explain the different response behaviors for the electrophysiological and the vascular response is addressed in the discussion section. This issue will be discussed in the light of the differentiation between evoked potentials and spontaneous background activity in the EEG measurements.

\section{Discussion}

Coupling between stimulus-induced electrophysiological and cerebrovascular changes allows for high-resolution functional mapping down to columnar (Cheng et al., 2001) and layerspecific topography (Pfeuffer et al., 2004). However, response modalities exhibit fundamental discrepancies. Because of a much longer latency, brief stimuli elicit a sluggish vascular response peaking after stimulus cessation (Martindale et al., 2003); oxygen demand generated by neuronal activity is overcompensated by a disproportionally large vascular response (Fox and Raichle, 1986). To explain the mismatch between oxygen consumption and blood flow, three major theories have been proposed: (1) Glucose uptake and metabolism determines blood flow (Frahm et al., 1996; Magistretti and Pellerin, 1996). (2) Limited oxygen diffusion to tissue necessitates an overly large increase in blood flow (Buxton and Frank, 1997). (3) Finally, a dominant role of neurotransmitter or interneuron links between the "functional unit" and the vessels has been highlighted (Attwell and Iadecola, 2002). Not mutually exclusive, theories are still controversial. The disproportionally large oxygen supply, however, constitutes the most powerful imaging signal, i.e., BOLD contrast reflecting decreases in [deoxy-Hb]. Temporal latency is efficiently dealt with by models of the "hemodynamic response function" (Friston et al., 1995).

Here, we highlight another discrepancy as yet not fully addressed. If neuronal networks encode information by synchronization of spontaneous oscillations (Gray et al., 1989; Fries et al., 2001), such neuronal processing may require neither more energy nor additional synaptic signaling. To test for hence predicted dissociations between evoked potential and vascular response, we investigated (1) ssVEP, (2) variation of $\alpha$-power, and (3) the hemodynamic responses to parametrically varied frequency of a flicker-light stimulation. We find a discontinuous maximum of the ssVEP and $\alpha$-power when stimulating at the individual $\alpha$-frequency. The oxygenation response does not show such a peak but steadily decreases in amplitude with stimulation frequencies $>8 \mathrm{~Hz}$. This indicates that the evoked potential is composed of several electrophysiological processes, including the synchronization of spontaneous oscillations. A comparison between evoked potentials and a vascular response must therefore respect both the summation of evoked neuronal signaling and the synchronization of spontaneous rhythmic activity, the latter potentially not reflected by a larger vascular response. In addition to this discontinuity of frequency dependence in the electrophysiological parameters assessed, the ssVEP steadily decreases with increasing stimulus rate. $\alpha$-Power decreases up to the IAF, showing a small increase for higher frequencies. Thus, neither of the frequency dependencies easily predicts the vascular response, which was maximal at a $7-8 \mathrm{~Hz}$ stimulation frequency. Lower stimulation rates elicit a smaller [deoxy-Hb]-response, whereas higher frequencies slightly attenuate the vascular response. This corresponds to reports on frequency dependence for visual stimulation with PET and fMRI (Fox and Raichle, 1985; Kwong et al., 1992; Thomas and Menon, 1998; Ozus et al., 2001; Hagenbeek et al., 2002). Although the experimental designs differed in stimulus conditions, stimulus intensity, and the frequency range tested, response saturation mostly at $8 \mathrm{~Hz}$ is agreed on. Higher frequencies yield a small decrease or no further amplitude modulation (Ozus et al., 2001). The reason for a peak response at $8 \mathrm{~Hz}$ remains unclear. Fox and Raichle (1985) discussed the "BrückeBartley effect," predicting the largest population of cortical neurons to respond to frequency adjusted to the "activity-recovery cycle" in the retina-cortex pathway (Grüsser and Creutzfeldt, 1957; Bartley, 1968). Although ssVEP is a coarse measure of integrated electrophysiological activity, the expected maximal neuronal recruitment cannot explain a local minimum of the evoked vascular response. Figure $4 a$, however, demonstrates a local minimum of ssVEP at $7 \mathrm{~Hz}$. Thus, we cannot confirm the maximal hemodynamic response at $8 \mathrm{~Hz}$ to simply reflect the BrückeBartley effect.

Alternatively, our results suggest that the hemodynamic response will also depend on the modulation of background activity (Brookes et al., 2005). Electrophysiologically, $\alpha$-rhythm has long been considered to indicate an "idling state" desynchronized by incoming visual stimuli (Berger, 1929; Fries et al., 2001). Because photic driving does not completely abolish $\alpha$-activity (Adrian and Matthews, 1934), we also assessed the modulation of $\alpha$-power at all stimulation frequencies. Assuming an inverse relationship between $\alpha$-power and BOLD contrast in the visual cortex (Singh et al., 2002; Laufs et al., 2003), we expected a correlation between the amplitude of the [deoxy-Hb] response and $\alpha$-power (Moosmann et al., 2003). Indeed, we find that $\alpha$-power and [deoxy-Hb] decrease with increasing stimulus frequency up to $7-8 \mathrm{~Hz}$. There is a discontinuity of the $\alpha$-power amplitude around the IAF to slightly increase with flicker frequencies beyond $12 \mathrm{~Hz}$. Thus, our data provide evidence for the view that $\alpha$-power modulation will influence the vascular response also during ongoing stimulation (Brookes et al., 2005).

The resonance between ssVEP and $\alpha$-power at the IAF deserves additional consideration. Typical VEP assessment assumes that background activity is independent of the EPs. Event-locked averaging recovers the VEP from a background, which is dominated by oscillations in the $\alpha$-range over the occipital lobe. How- 
ever, evidence has been provided that ongoing activity is altered in amplitude and/or phase by incoming stimuli. Stimulusinduced phase-locking of the $\alpha$-rhythm has been shown to substantially contribute to the generation of VEPs (Makeig et al., 2002). Also, stimulus-driven modulation of locked and unlocked $\alpha-, \theta-$, and $\gamma$-bands have been reported (Schurmann and Basar, 1994; Basar et al., 1998; Pfurtscheller, 2001; Woertz et al., 2004). Thus, beyond clinical practice, it is appropriate to consider ongoing activity and evoked potentials, sharing a common corticothalamic pathway, mutually dependent (Lopes da Silva et al., 1980; Chatila et al., 1993). In our study, we find a resonance between the $\alpha$-power and evoked potentials at the IAF. Frequency domain analysis of $\alpha$-power introduces the problem that stimulus-locked evoked potentials and $\alpha$-power will both peak at $10-11 \mathrm{~Hz}$. Therefore, we attenuated the stimulus-evoked contribution by subtraction of the mean evoked potential before the spectral analysis. Such an additive model of the "reproducible response," estimated by trial averaging and the "ongoing activity" to predict the measured activity was also proposed by Arieli et al. (1996). The persisting resonance of both VEP and $\alpha$-power at the IAF indicates phase locking and augmentation of the background $\alpha$-band activity. In line with previous publications (Herrmann, 2001), there is additional evidence for phase locking because the simultaneously assessed hemodynamic response does not show a local maximum at the IAF. The reasoning is as follows: although mechanisms of coupling are still controversial, there is ample evidence that integrated afferent signaling may best predict the hemodynamic response (Lauritzen, 2001). Even inhibitory synaptic activity evokes increases in rCBF (Mathiesen et al., 1998); hence, any increase in cortical processing should evoke an increased vascular response.

Our result allows for two explanations: (1) phase-locking between $\alpha$-rhythm and equifrequent stimulation will not require a substantial recruitment of cortical processing; (2) inhibitory corticocortical connections are blocked when stimulating at the IAF.

Although we favor the first explanation, it is impossible to disprove the second potential interpretation. Metabolic demand and ensuing vascular response caused by inhibition in a region are highly controversial (Tagamets and Horwitz, 2001). Metabolically, GABA cycling has been shown to not increase glucose uptake in astrocytes (Chatton et al., 2003), and BOLD contrast has been considered insensitive to inhibition (Waldvogel et al., 2000). However, rCBF increases have been demonstrated in response to inhibition (Mathiesen et al., 1998). Theoretically, blocking GABAergic neurons might decrease $\mathrm{rCBF}$, which might compensate for the increase in $\mathrm{rCBF}$ attributable to excitatory neuronal activity. This could explain the insensitivity of the vascular response to the electrophysiological resonance. Because inhibition is more efficient as a result of the strategic positioning of inhibitory neurons (Koos and Tepper, 1999), our prediction is that even complete blocking of GABAergic neurons will cause a net increase in metabolic demand and $\mathrm{rCBF}$, yielding a larger washout of [deoxy-Hb]. However, noninvasive approaches such as those used here will not be able to prove or disprove such a prediction. In brief, by comparing the amplitude of an evoked potential with the corresponding vascular response, we find clear deviations from a more or less linear dependency for a parametrically varied stimulus. This conflicts with a notion of a simple translation of the evoked potential into changes in hemodynamics (Ngai et al., 1999; Arthurs et al., 2000).

In the face of the accumulating evidence for substantial nonlinearities of neurovascular coupling, it may seem advisory to rather refrain from any inference as to neuronal computations based on vascular response mapping. One might indulge in a reappraisal of electrophysiology, providing direct precisely timed information on the most relevant structure, the neuron. However, beyond the "inverse problem" in scalp recordings, even invasive studies have not solved the question of whether a stimulus will be represented by a specific excitation/inhibition pattern or whether synchronization of spontaneous oscillations are the correlate of a stimulus-evoked percept or whether both holds true (Stryker, 1989; Pareti and De Palma, 2004). Our study demonstrates that the comprehensive view of either side of the coin can provide answers to these very questions.

\section{References}

Adrian E, Matthews B (1934) The Berger rhythm: potential changes from the occipital lobes in man. Brain 57:355-384.

Arieli A, Sterkin A, Grinvald A, Aertsen A (1996) Dynamics of ongoing activity: explanation of the large variability in evoked cortical responses. Science 273:1868-1871.

Arthurs OJ, Williams EJ, Carpenter TA, Pickard JD, Boniface SJ (2000) Linear coupling between functional magnetic resonance imaging and evoked potential amplitude in human somatosensory cortex. Neuroscience 101:803-806.

Attwell D, Iadecola C (2002) The neural basis of functional brain imaging signals. Trends Neurosci 25:621-625.

Bartley SH (1968) Temporal features of input as crucial factors in vision. Contrib Sens Physiol 3:81-124.

Basar E, Rahn E, Demiralp T, Schurmann M (1998) Spontaneous EEG theta activity controls frontal visual evoked potential amplitudes. Electroencephalogr Clin Neurophysiol 108:101-109.

Berger H (1929) Über das Elektrenkephalogramm des Menschen. Arch Psychiatr Nervenkr 87:527-570.

Brookes MJ, Gibson AM, Hall SD, Furlong PL, Barnes GR, Hillebrand A, Singh KD, Holliday IE, Francis ST, Morris PG (2005) GLMbeamformer method demonstrates stationary field, alpha ERD and gamma ERS co-localisation with $\mathrm{AMRI}$ BOLD response in visual cortex. NeuroImage 26:302-308.

Buxton RB, Frank LR (1997) A model for the coupling between cerebral blood flow and oxygen metabolism during neural stimulation. J Cereb Blood Flow Metab 17:64-72.

Chatila M, Milleret C, Rougeul A, Buser P (1993) Alpha rhythm in the cat thalamus. C R Acad Sci III 316:51-58.

Chatton JY, Pellerin L, Magistretti PJ (2003) GABA uptake into astrocytes is not associated with significant metabolic cost: implications for brain imaging of inhibitory transmission. Proc Natl Acad Sci USA 100:12456-12461.

Cheng K, Waggoner RA, Tanaka K (2001) Human ocular dominance columns as revealed by high-field functional magnetic resonance imaging. Neuron 32:359-374.

Cope M, Delpy DT (1988) System for long-term measurement of cerebral blood and tissue oxygenation on newborn infants by near infra-red transillumination. Med Biol Eng Comput 26:289-294.

Fox PT, Raichle ME (1985) Stimulus rate determines regional brain blood flow in striate cortex. Ann Neurol 17:303-305.

Fox PT, Raichle ME (1986) Focal physiological uncoupling of cerebral blood flow and oxidative metabolism during somatosensory stimulation in human subjects. Proc Natl Acad Sci USA 83:1140-1144.

Frahm J, Kruger G, Merboldt KD, Kleinschmidt A (1996) Dynamic uncoupling and recoupling of perfusion and oxidative metabolism during focal brain activation in man. Magn Reson Med 35:143-148.

Fries P, Reynolds JH, Rorie AE, Desimone R (2001) Modulation of oscillatory neuronal synchronization by selective visual attention. Science 291:1560-1563.

Friston KJ, Holmes AP, Poline JB, Grasby PJ, Williams SC, Frackowiak RS, Turner R (1995) Analysis of fMRI time-series revisited. NeuroImage 2:45-53.

Goldman RI, Stern JM, Engel Jr J, Cohen MS (2002) Simultaneous EEG and fMRI of the alpha rhythm. NeuroReport 13:2487-2492.

Gray CM, Konig P, Engel AK, Singer W (1989) Oscillatory responses in cat visual cortex exhibit inter-columnar synchronization which reflects global stimulus properties. Nature 338:334-337.

Grüsser OJ, Creutzfeldt O (1957) Neurophysiological basis of Brucke- 
Bartley effect; maxima of impulse frequency of retinal and cortical neurons in flickering light of middle frequency. Pflügers Arch 263:668-681.

Hagenbeek RE, Rombouts SA, van Dijk BW, Barkhof F (2002) Determination of individual stimulus-response curves in the visual cortex. Hum Brain Mapp 17:244-250.

Herrmann CS (2001) Human EEG responses to 1-100 Hz flicker: resonance phenomena in visual cortex and their potential correlation to cognitive phenomena. Exp Brain Res 137:346-353.

Hewson-Stoate N, Jones M, Martindale J, Berwick J, Mayhew J (2005) Further nonlinearities in neurovascular coupling in rodent barrel cortex. NeuroImage 24:565-574.

Kleinschmidt A, Obrig H, Requardt M, Merboldt KD, Dirnagl U, Villringer A, Frahm J (1996) Simultaneous recording of cerebral blood oxygenation changes during human brain activation by magnetic resonance imaging and near-infrared spectroscopy. J Cereb Blood Flow Metab 16:817-826.

Kohl-Bareis M, Obrig H, Steinbrink J, Malak J, Uludag K, Villringer A (2002) Noninvasive monitoring of cerebral blood flow by a dye bolus method: separation of brain from skin and skull signals. J Biomed Opt 7:464-470.

Koos T, Tepper JM (1999) Inhibitory control of neostriatal projection neurons by GABAergic interneurons. Nat Neurosci 2:467-472.

Kwong KK, Belliveau JW, Chesler DA, Goldberg IE, Weisskoff RM, Poncelet BP, Kennedy DN, Hoppel BE, Cohen MS, Turner R (1992) Dynamic magnetic resonance imaging of human brain activity during primary sensory stimulation. Proc Natl Acad Sci USA 89:5675-5679.

Laufs H, Kleinschmidt A, Beyerle A, Eger E, Salek-Haddadi A, Preibisch C, Krakow K (2003) EEG-correlated fMRI of human alpha activity. NeuroImage 19:1463-1476.

Lauritzen M (2001) Relationship of spikes, synaptic activity, and local changes of cerebral blood flow. J Cereb Blood Flow Metab 21:1367-1383.

Logothetis NK, Pauls J, Augath M, Trinath T, Oeltermann A (2001) Neurophysiological investigation of the basis of the fMRI signal. Nature 412:150-157.

Lopes da Silva FH (2004) Biophysical aspects of EEG and magnetoencephalogram generation. In: EEG: basic principles, clinical applications, and related fields (Niedermeyer E, Lopes da Silva FH, eds), pp 107-125. Philadelphia: Lippincott Williams \& Wilkins.

Lopes da Silva FH, Vos JE, Mooibroek J, Van Rotterdam A (1980) Relative contributions of intracortical and thalamo-cortical processes in the generation of alpha rhythms, revealed by partial coherence analysis. Electroencephalogr Clin Neurophysiol 50:449-456.

Magistretti PJ, Pellerin L (1996) Cellular bases of brain energy metabolism and their relevance to functional brain imaging: evidence for a prominent role of astrocytes. Cereb Cortex 6:50-61.

Makeig S, Westerfield M, Jung TP, Enghoff S, Townsend J, Courchesne E, Sejnowski TJ (2002) Dynamic brain sources of visual evoked responses. Science 295:690-694.

Martindale J, Mayhew J, Berwick J, Jones M, Martin C, Johnston D, Redgrave P, Zheng Y (2003) The hemodynamic impulse response to a single neural event. J Cereb Blood Flow Metab 23:546-555.

Mathiesen C, Caesar K, Akgoren N, Lauritzen M (1998) Modification of activity-dependent increases of cerebral blood flow by excitatory synaptic activity and spikes in rat cerebellar cortex. J Physiol (Lond) 512:555-566.

Mentis MJ, Alexander GE, Grady CL, Horwitz B, Krasuski J, Pietrini P, Strassburger T, Hampel H, Schapiro MB, Rapoport SI (1997) Frequency variation of a pattern-flash visual stimulus during PET differentially activates brain from striate through frontal cortex. NeuroImage 5:116-128.

Mitzdorf U (1985) Current source-density method and application in cat cerebral cortex: investigation of evoked potentials and EEG phenomena. Physiol Rev 65:37-100.
Moosmann M, Ritter P, Krastel I, Brink A, Thees S, Blankenburg F, Taskin B, Obrig H, Villringer A (2003) Correlates of alpha rhythm in functional magnetic resonance imaging and near infrared spectroscopy. NeuroImage 20:145-158.

Ngai AC, Jolley MA, D’Ambrosio R, Meno JR, Winn HR (1999) Frequencydependent changes in cerebral blood flow and evoked potentials during somatosensory stimulation in the rat. Brain Res 837:221-228.

Niessing J, Ebisch B, Schmidt KE, Niessing M, Singer W, Galuske RA (2005) Hemodynamic signals correlate tightly with synchronized gamma oscillations. Science 309:948-951.

Obrig H, Villringer A (2003) Beyond the visible-imaging the human brain with light. J Cereb Blood Flow Metab 23:1-18.

Odom JV, Bach M, Barber C, Brigell M, Marmor MF, Tormene AP, Holder GE, Vaegan (2004) Visual evoked potentials standard. Doc Ophthalmol 108:115-123.

Ozus B, Liu HL, Chen L, Iyer MB, Fox PT, Gao JH (2001) Rate dependence of human visual cortical response due to brief stimulation: an eventrelated fMRI study. Magn Reson Imaging 19:21-25.

Pareti G, De Palma A (2004) Does the brain oscillate? The dispute on neuronal synchronization. Neurol Sci 25:41-47.

Pastor MA, Artieda J, Arbizu J, Valencia M, Masdeu JC (2003) Human cerebral activation during steady-state visual-evoked responses. J Neurosci 23:11621-11627.

Pfeuffer J, Merkle H, Beyerlein M, Steudel T, Logothetis NK (2004) Anatomical and functional MR imaging in the macaque monkey using a vertical large-bore 7 tesla setup. Magn Reson Imaging 22:1343-1359.

Pfurtscheller G (2001) Functional brain imaging based on ERD/ERS. Vision Res 41:1257-1260.

Schurmann M, Basar E (1994) Topography of alpha and theta oscillatory responses upon auditory and visual stimuli in humans. Biol Cybern 72:161-174.

Sewards TV, Sewards MA (1999) Alpha-band oscillations in visual cortex: part of the neural correlate of visual awareness? Int J Psychophysiol 32:35-45.

Singh KD, Barnes GR, Hillebrand A, Forde EM, Williams AL (2002) Taskrelated changes in cortical synchronization are spatially coincident with the hemodynamic response. NeuroImage 16:103-114.

Steinbrink J, Villringer A, Kempf F, Haux B, Boden S, Obrig H (2006) Illuminating the BOLD-signal: combined fMRI-fNIRS studies. Magn Reson Imaging, in press.

Stryker MP (1989) Cortical physiology. Is grandmother an oscillation? Nature 338:297-298.

Tagamets MA, Horwitz B (2001) Interpreting PET and fMRI measures of functional neural activity: the effects of synaptic inhibition on cortical activation in human imaging studies. Brain Res Bull 54:267-273.

Thomas CG, Menon RS (1998) Amplitude response and stimulus presentation frequency response of human primary visual cortex using BOLD EPI at 4 T. Magn Reson Med 40:203-209.

Villringer A, Dirnagl U (1995) Coupling of brain activity and cerebral blood flow: basis of functional neuroimaging. Cerebrovasc Brain Metab Rev 7:240-276.

Waldvogel D, van GP, Muellbacher W, Ziemann U, Immisch I, Hallett M (2000) The relative metabolic demand of inhibition and excitation. Nature 406:995-998.

Woertz M, Pfurtscheller G, Klimesch W (2004) Alpha power dependent light stimulation: dynamics of event-related (de)synchronization in human electroencephalogram. Brain Res Cogn Brain Res 20:256-260. 